\title{
Fonte alimentar sangüínea e a peridomiciliação de Lutzomyia longipalpis (Lutz \& Neiva, 1912) (Psychodidae, Phlebotominae)
}

\author{
Blood feeding sources and peridomiciliation \\ of Lutzomyia longipalpis (Lutz \& Neiva, 1912) \\ (Psychodidae, Phlebotominae)
}

Flávio de Oliveira Passos Dias 1

Elias Seixas Lorosa 2

José Manuel Macário Rebêlo 1

\footnotetext{
1 Laboratório de

Entomologia e Vetores,

Departamento de Patologia,

Universidade Federal

do Maranhão.

Praça Madre Deus 2,

São Luís, MA

65025-560, Brasil.

flavioopd@bol.com.br

macariorebelo@uol.com.br

2 Laboratório Nacional

e Internacional de

Referência em Taxonomia

de Triatomíneos,

Departamento de

Entomologia, Instituto

Oswaldo Cruz.

Av. Brasil 4365,

Rio de Janeiro, $R J$

21045-900, Brasil.
}

\begin{abstract}
A precipitin test was employed to study the alimentary tract content of Lutzomyia longipalpis in the intra-and peridomiciliary environments in the municipality of Raposa, Maranhão State, a transmission area for visceral leishmaniasis or kala azar. Out of 2,240 female sandflies captured, 547 (24.4\%) had fed on vertebrate blood, with the following proportions: avian (87.9\%); rodent (47.2\%); human (42.4\%); canine (27.6\%); opossum (26.6\%); and equine (22.5\%). Based on a survey of 120 human dwellings, chickens were found to be the most common domestic animals in the peridomicile (28.3\%), followed by dogs (21.7\%), cats (17.5\%), donkeys (13.3\%), pigeons (7.5\%), rabbits (3.3\%), ducks (3.3\%), and horses, mallards, and pigs (1.7\% each). Synanthropic animals included opossums (39.3\%), followed by rats (37.9\%), bats (14.3\%), raccoons (3.6\%), foxes (2.1\%), snakes (1.4\%), and frogs (1.4\%). The peridomiciliary presence of domestic and synanthropic animals as well as sandflies that had fed on human, opossum, and canid blood supports the hypothesis that kala azar transmission has been taking place in the anthropic environment in the municipality of Raposa.
\end{abstract}

Key words Visceral Leishmaniasis; Psychodidae; Vector Control

Resumo Estudou-se por meio da reação da precipitina, o conteúdo estomacal de Lutzomyia longipalpis nos ambientes intradomiciliar e peridoméstico, no Município de Raposa, Maranhão, área de transmissão de leishmaniose visceral ou "calazar". De 2.240 fêmeas capturadas, 547 $(24,4 \%)$ estavam alimentadas com sangue de vertebrados nas proporções que seguem: ave (87,9\%); roedor (47,2\%); humano (42,4\%), cão (27,6\%); mucura (26,6\%) e eqüino (22,5\%). A investigação levada a efeito em 120 habitações confirmou a galinha como o animal doméstico mais comum no ambiente peridoméstico (28,3\%), seguido pelo cão (21,7\%), gato (17,5\%), jumento (13,3\%), pombo (7,5\%), coelho (3,3\%) e pato (3,3\%); enquanto o cavalo, marreco e porco representaram, cada um, 1,7\%. Entre os animais sinantrópicos, a mucura foi a mais citada naquele ambiente (39,3\%), seguida pelo rato (37,9\%), morcego (14,3\%) guaxinim (3,6\%), raposa (2,1\%), cobra (1,4\%) e sapo (1,4\%). A presença no peridomicílio de animais domésticos e sinantrópicos e o encontro de flebótomos alimentados, ao mesmo tempo, com sangue humano, de mucura e de canídeos, corroboram a hipótese de que a transmissão do calazar esteja ocorrendo realmente no ambiente antrópico, no Município de Raposa.

Palavras-chave Leishmaniose Visceral; Psychodidae; Controle de Vetores 


\section{Introdução}

Os flebotomíneos, assim como muitos outros dípteros hematófagos, necessitam de suprimentos de carboidratos que, na natureza, adquirem diretamente da seiva de plantas, néctar (Alexander \& Usma, 1994), secreções de afídeos e frutas maduras (Cameron et al., 1995). Para as fêmeas, esses requerimentos são utilizados como complemento na alimentação sangüínea (Magnarelli \& Modi, 1988; Van Handel, 1984). Aliás, a hematofagia é um hábito exclusivo das fêmeas, que necessitam do sangue tão somente para a maturação dos ovários e o obtém sugando diversos vertebrados (mamíferos, aves, répteis e anfíbios).

O estudo do conteúdo estomacal de insetos hematófagos é de grande significado ecológico e epidemiológico. Além de permitir descobrir a identidade dos hospedeiros sobre os quais os flebotomíneos se alimentam, por ocasião do repasto sangüíneo, pode fornecer subsídios para a indicação de reservatórios potenciais de leishmânias e o papel protetor que certos animais poderiam desempenhar em relação ao homem em área de transmissão destes parasitas (Ferreira, 1945).

Atualmente, é conhecida uma variedade de animais que já foram identificados como hospedeiros alimentares de flebotomíneos (Boreham, 1975, apud Lainson \& Shaw, 1979; Christensen et al., 1982; Tesh et al., 1971), inclusive infectados por alguma espécie de Leishmania. Estudos desta natureza utilizando Lutzomyia longipalpis foram levados a efeito na Colômbia (Morrison et al., 1993) e na Amazônia brasileira (Quinnell et al., 1992). A maior parte dos dados disponíveis na literatura mostra que o caráter oportunista parece predominar na alimentação desses insetos que podem sugar ampla variedade de vertebrados.

Enfim, o conhecimento dos hábitos alimentares de espécies de flebotomíneos e de suas fontes sangüíneas tem sido muito útil ao aperfeiçoamento dos conhecimentos sobre a epidemiologia das leishmânias e, neste sentido, tem norteado as atividades de controle e vigilância. Portanto, procurou-se estudar a fonte alimentar de L. longipalpis e a importância dos animais domésticos e sinantrópicos na manutenção do vetor no ambiente peridoméstico. Este trabalho é o primeiro dessa natureza realizado no Maranhão.

\section{Material e métodos}

O estudo foi realizado na Vila Bom Viver, Município de Raposa, que se situa na Ilha de São Luís, ao norte do Estado do Maranhão (Figura 1). Abrange uma área de $75 \mathrm{~km}^{2}$, limitando-se ao norte e oeste com o Oceano Atlântico, ao sul e leste com o Município de Paço do Lumiar. O clima é quente e úmido, com temperatura média anual em torno de $28^{\circ} \mathrm{C}$. Apresenta duas estações bem definidas, uma de estiagem de julho a novembro, e outra chuvosa de dezembro a junho (IBGE, 1996). A estação chuvosa concentra, em média, $94 \%$ do total anual das chuvas, apresentando índices pluviométricos mais elevados nos meses de abril a junho. Durante a estiagem, a deficiência hídrica se faz sentir principalmente nos meses de outubro a novembro. A precipitação pluviométrica anual é de $1.900 \mathrm{~mm}$. A altitude média é de $5 \mathrm{~m}$ nas partes mais baixas e $15 \mathrm{~m}$ nas dunas e próximo aos córregos e vales, mas grande parte de sua área está submersa em água salgada. O ponto mais alto corresponde ao morro da Ponta do Farol, que mede 45m de altitude, onde fica o Farol do Araçagi.

A hidrografia do município é constituída principalmente por praias, lagos e pequenos rios, como o rio Pimenta. Destacam-se as praias do Araçagi, Raposa, Carimã, Curupu e Belizário, onde se localiza o terminal pesqueiro constituído por bocas de rios.

De acordo com o IBGE (1996), o Município de Raposa tem uma população de 15.075 habitantes, $7.794(51,7 \%)$ são do sexo masculino e $7.281(48,3 \%)$ do sexo feminino. Aproximadamente $38 \%$ da população é representada por pessoas com idade entre 0 e 14 anos. A principal atividade local é a pesca e a manufatura do artesanato de palha.

Foram selecionados dois locais para a captura dos flebótomos (Figura 2), um ocupado há doze anos (Figura 2a), correspondendo à periferia de um núcleo habitacional; e outro com características rurais, ocupado por invasão há quatro anos (Figura 2b). A distância entre os dois locais é de aproximadamente um quilômetro.

\section{Procedimentos}

No local A, a residência era de alvenaria e servida de todos os benefícios urbanos, porém, com amplo peridomicílio, contendo abrigos de coelhos, galinhas, patos, ovelhas, porcos e cavalos, além da presença de cão e gato. Estes abrigos distavam cerca de dez a trinta metros da residência (Figura 2a). Neste local, uma ar- 
madilha CDC foi instalada a doze metros da residência, entre os abrigos de animais domésticos e outra no dormitório da residência (Figura 2a).

No local B, a residência era de taipa e isolada entre áreas abertas e capoeiras baixas. No peridomicílio (quintal), que continha galinha, jumento, cão e gato, uma armadilha CDC foi instalada a quatro metros da residência, entre o galinheiro e o estábulo, e outra foi instalada no dormitório da habitação (Figura 2b). Os abrigos de animais distavam cerca de três a seis metros da residência.

Os flebótomos foram capturados durante dois anos, de julho de 1999 a junho de 2001, duas vezes por mês, no horário das 18 às 6 horas. Os exemplares retidos nas armadilhas foram transferidos para câmaras mortíferas à base de acetato de etila e transportados a seco para o laboratório. Todos os flebótomos coletados foram transferidos para placas de Petri, onde foram submetidos à triagem sob estereomicroscópio, separando-se os flebótomos dos demais insetos, os machos das fêmeas. Uma vez separados, cada lote foi acondicionado em freezer a $20^{\circ} \mathrm{C}$, em tubos de plásticos tipo Eppendorf, etiquetados com os seguintes dados: hora, local, armadilha e coletor.

O teste de precipitina foi aplicado ao conteúdo intestinal de fêmeas de flebótomos previamente alimentadas com sangue de vertebrados. A técnica foi executada com pequenas quantidades dos reagentes para facilitar a leitura, e a identificação foi realizada com algumas modificações, visto que foi coletado o tubo digestivo dos flebótomos e, o conteúdo estomacal foi triturado em salina a $0,85 \%$. Este macerado foi deixado por 12 horas à temperatura entre $4{ }^{\circ} \mathrm{C}$ e $8{ }^{\circ} \mathrm{C}$ e, logo após, centrifugado por 5 minutos a $1.500 \mathrm{rpm}$, e o sobrenadante confrontando com os anti-soros de humano, cão, gato, cavalo, cabra, porco, roedor, mucura, tatu, carneiro, ave, lagarto e sapo.

Durante o estudo foram visitadas 120 moradias em que se registrou a presença de animais domésticos e se questionou acerca de visitas de animais sinantrópicos no peridomicílio e dentro das casas. Fizeram-se, ainda, medições da distância entre os abrigos de animais domésticos e as habitações humanas, observando o tipo e a qualidade que apresentavam.

\section{Resultados}

Foram capturadas 2.240 fêmeas de L. Longipalpis, sendo $84 \%$ no ambiente peridoméstico e $16 \%$ no intradomicílio. Do total de fêmeas cap-
Figura 1

Mapa da Ilha de São Luís, Estado do Maranhão, mostrando o povoado de Bom Viver, Município da Raposa, local deste estudo.
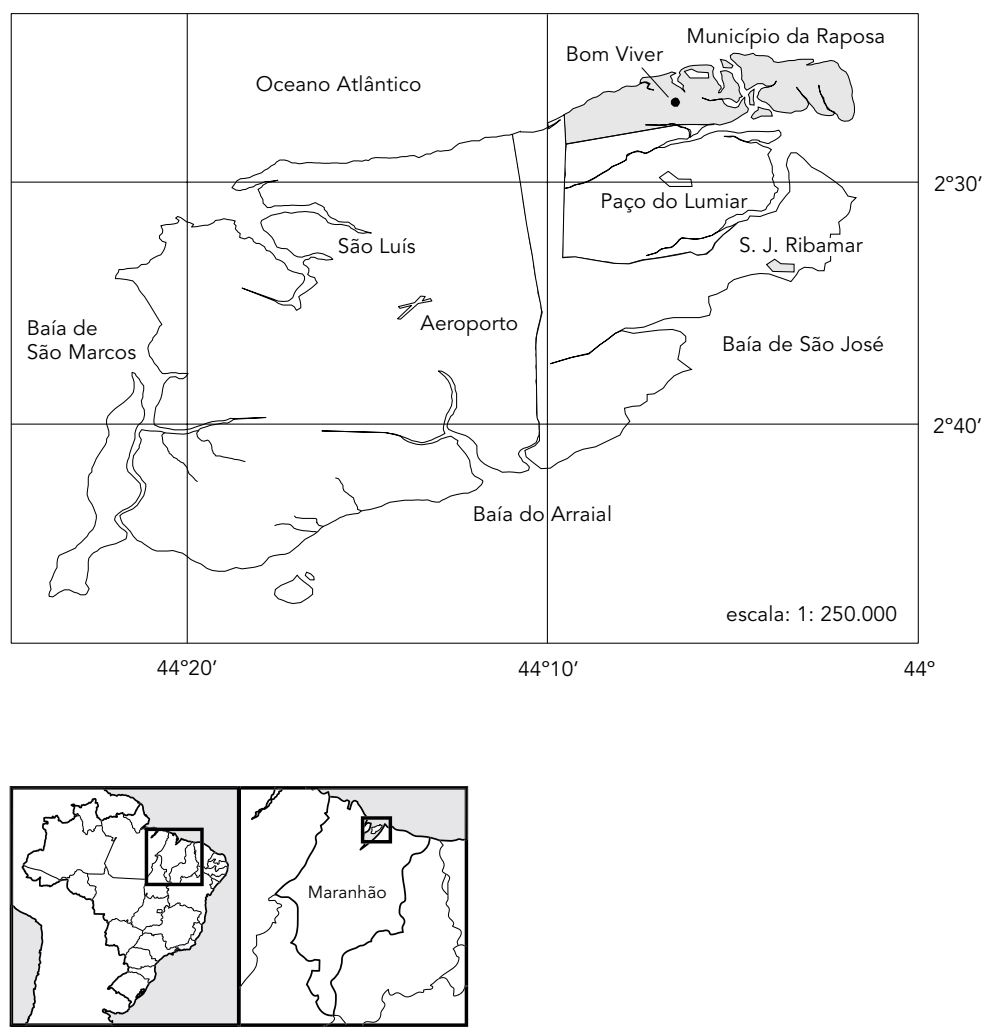

turadas, $1.693(75,6 \%)$ não apresentaram vestígios de sangue no conteúdo intestinal, enquanto 547 estavam alimentadas com sangue, representando $24,4 \%$ do total de espécimes amostrados. Os flebótomos alimentados haviam sugado sangue de mais de um hospedeiro, tendo sido encontradas as seguintes combinações, com respectivos percentuais: aves e roedor (11\%); ave e humano $(20,1 \%)$; ave e mucura $(7,8 \%)$; ave, cão e jumento $(7,4 \%)$; ave, cão e roedor $(10 \%)$; ave, humano e mucura $(9,4 \%)$; ave, humano e jumento $(8,1 \%)$; ave, roedor e mucura $(9,2 \%)$; ave, cão, humano e roedor (5\%); jumento e roedor $(6,9 \%)$; e, por fim, cão e roedor $(5,1 \%)$ (Tabela 1).

Estas combinações se deram em virtude das análises da reação da precipitina terem sido realizadas em pool (lotes). Somente nas últimas duas combinações não se perceberam o envolvimento de aves. Este grupo de animal foi o mais procurado, sendo sugado por $87,9 \%$ dos 
Figura 2

Esquema mostrando os locais onde as armadilhas $\operatorname{CDC}(\bullet)$ foram instaladas, na área rural (Figura 2a) e periurbana (Figura 2b), e a relativa distância (setas) entre os abrigos animais e a residência. As linhas interrompidas correspondem ao provável trajeto percorrido pelos animais sinantrópicos.

\section{Figura 2a}

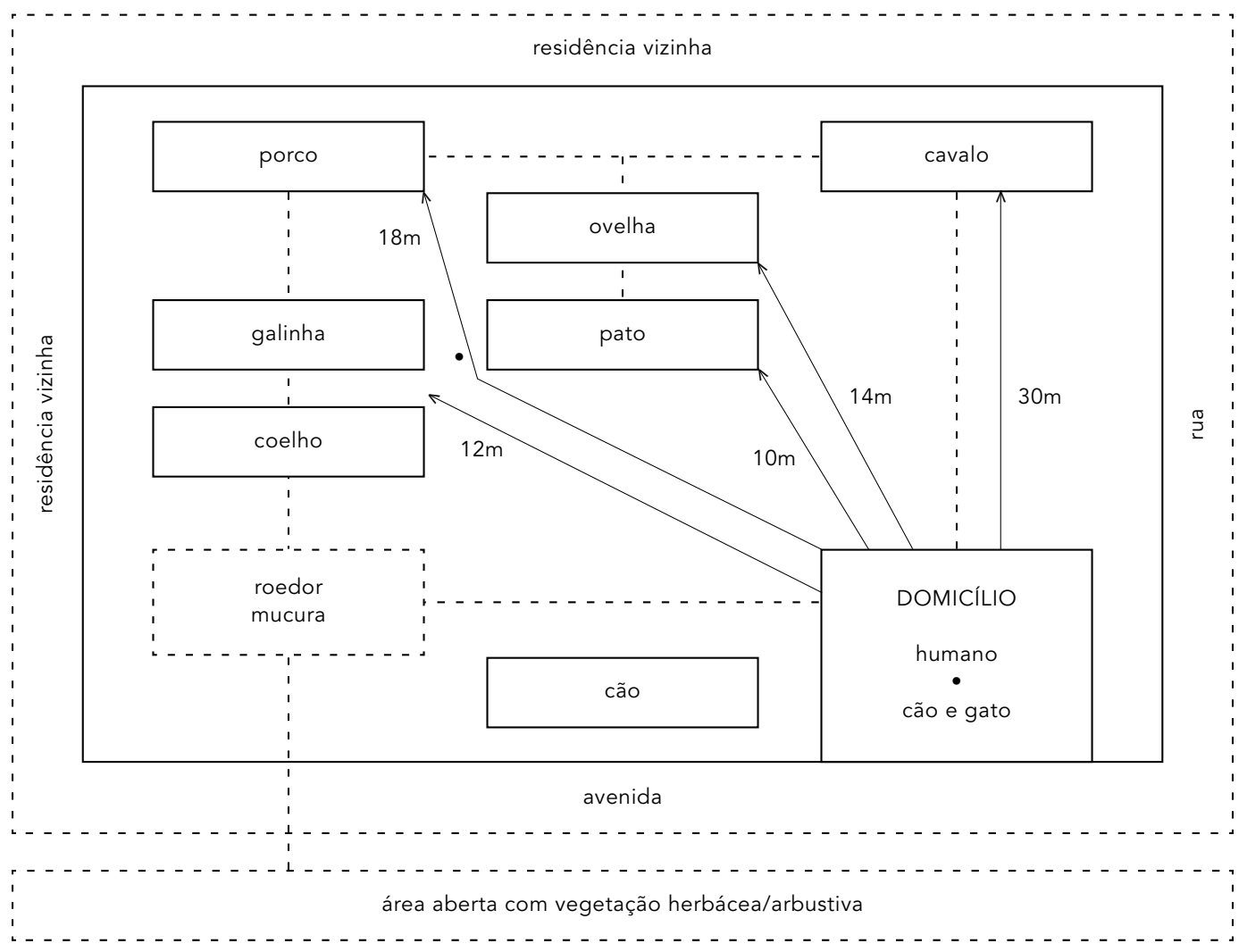

\section{Figura 2b}

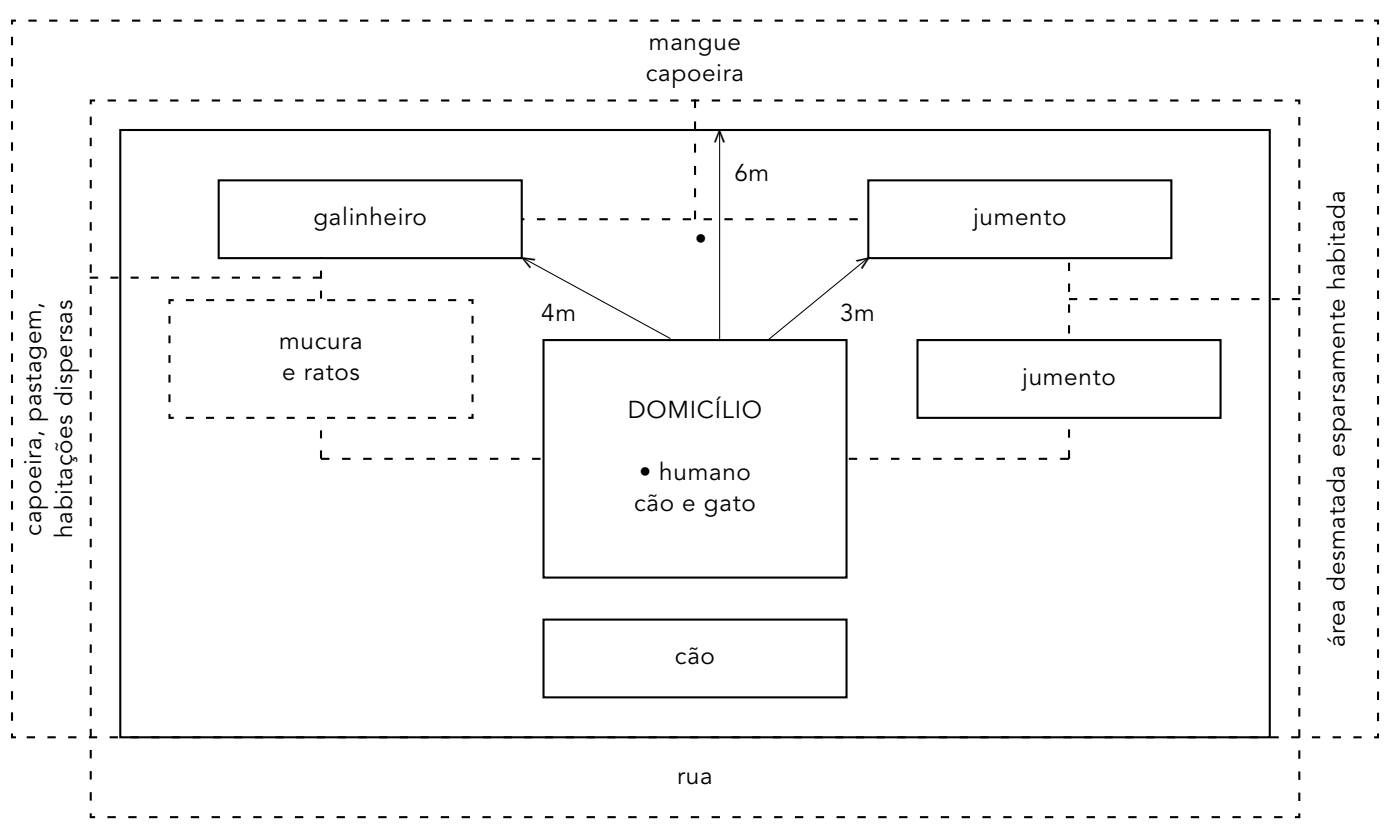


flebótomos alimentados. As proporções dos flebótomos que sugaram o sangue de outros vertebrados foram as que seguem: roedor $(47,2 \%)$; humano (42,4\%); cão (27,6\%); mucura (26,3\%); e eqüino (22,5\%) (Tabela 2$)$.

$\mathrm{O}$ inquérito sobre a existência de animais domésticos em 120 moradias confirmou a galinha como o vertebrado de maior densidade em cada residência e o mais comum na área estudada, pois estava presente em $28,3 \%$ das habitações investigadas (Figura 3a). Seguiram, na ordem de importância, o cão $(21,7 \%)$, gato $(17,5 \%)$, jumento $(13,3 \%)$, pombo $(7,5 \%)$, coelho $(3,3 \%)$, pato $(3,3 \%)$, enquanto o cavalo, marreco e porco representaram, cada um, $1,7 \%$.

Entre os animais sinantrópicos (Figura 3b), a mucura (Didelphis marsupialis) foi o animal citado pela comunidade local como aquele que mais freqüentava o ambiente peridoméstico $(39,3 \%)$, seguida pelo rato $(37,9 \%)$, morcego $(14,3 \%)$, guaxinim - Procyon cancrivorous $(3,6 \%)$, raposa-Cerdocyon thous $(2,1 \%)$, cobra $(1,4 \%)$ e sapo $(1,4 \%)$.

Ainda sobre o inquérito realizado junto à comunidade, verificou-se que a distância dos abrigos de animais domésticos para as habitações variaram de 1 a 2 metros, em $73,6 \%$ dos casos, 3 a $5 \mathrm{~m}(24,3 \%)$, e 6 a $10 \mathrm{~m}(2,1 \%)$. Verificou-se, também, que entre os animais que pernoitavam dentro de casa, cerca de $55 \%$ se acomodam na sala, enquanto $34,3 \%$ permaneciam no quarto, e $10,7 \%$, na cozinha.

\section{Discussão}

O estudo do conteúdo estomacal de inseto hematófago é uma alternativa a fim de se conhecerem os animais domésticos e sinantrópicos efetivamente utilizados como hospedeiros sangüíneos no ambiente antrópico. Com essa metodologia pôde-se constatar, neste estudo, o comportamento eclético de L. longipalpis, uma vez que sugou o sangue de aves e mamíferos domésticos e sinantrópicos, incluindo ainda na sua dieta, o sangue humano, considerando que os anti-soros utilizados para as análises restringiram-se apenas a esses grupos de vertebrados. Este caráter oportunista já foi verificado por outros autores (Morrison et al., 1993; Quinnell et al., 1992) e constitui um aspecto ecológico de grande relevância na epidemiologia do calazar.

A presença de animais domésticos e a possibilidade deles virem a participar como hospedeiros sangüíneos dos flebótomos podem favorecer a aproximação e a manutenção destes insetos no peridomicílio. Em nossas expe-

\begin{tabular}{|c|c|c|}
\hline \multicolumn{3}{|c|}{$\begin{array}{l}\text { Reações duplas, triplas e quádruplas das fontes } \\
\text { alimentares de Lutzomyia longipalpis (Lutz \& Neiva, } \\
\text { 1912), provenientes do Município de Raposa, } \\
\text { Maranhão, Brasil. }\end{array}$} \\
\hline \multirow[t]{2}{*}{ Combinações de hospedeiros } & \multicolumn{2}{|c|}{ Total } \\
\hline & $\mathrm{n}$ & $\%$ \\
\hline Ave e roedor & 60 & 11,0 \\
\hline Ave e humano & 110 & 20,1 \\
\hline Ave e mucura & 43 & 7,9 \\
\hline Ave, cão e jumento & 41 & 7,5 \\
\hline Ave, cão e roedor & 55 & 10,1 \\
\hline Ave, humano e mucura & 51 & 9,3 \\
\hline Ave, humano e jumento & 44 & 8,0 \\
\hline Ave, roedor e mucura & 50 & 9,1 \\
\hline Ave, cão, humano e roedor & 27 & 4,9 \\
\hline Jumento e roedor & 38 & 7,0 \\
\hline Cão e roedor & 28 & 5,1 \\
\hline Total & 547 & 100,0 \\
\hline
\end{tabular}

\begin{tabular}{llc} 
Tabela 2 & \multicolumn{3}{c}{ Treqüência de espécimens de Lutzomyia longipalpis } \\
(Lutz \& Neiva, 1912), alimentados com sangue de \\
cada grupo de vertebrado do Município de Raposa, \\
Maranhão, Brasil. \\
\multicolumn{3}{c}{ Total } \\
\hline Hospedeiros & \multicolumn{3}{c}{ n } \\
& 481 & 87,9 \\
\hline Ave & 258 & 47,2 \\
Roedor & 232 & 42,4 \\
Humano & 151 & 27,6 \\
Cão & 144 & 26,3 \\
Mucura & 123 & 22,5 \\
Eqüino & \multicolumn{3}{c}{} \\
\hline
\end{tabular}

riências na própria Vila Bom Viver, tem-se verificado que nas habitações que não possuem animais domésticos as capturas de flebótomos não são bem sucedidas.

$\mathrm{O}$ L. longipalpis tendeu a sugar mais o sangue de ave, porque este grupo de vertebrado apareceu em nove das onze reações duplas, triplas e quádruplas determinadas. Esta forte associação com o vetor pode resultar do fato da galinha constituir um dos animais domésticos mais atrativo e comum na Vila Bom Viver. Essa associação já foi observada no Ceará (Deane, 1956) e no Rio de Janeiro, Aguiar et al. (1987) 
Freqüência de habitações com animais domésticos (Figura 3a) e sinantrópicos (Figura 3b) encontrados no ambiente peridoméstico em Bom Viver, Município de Raposa, Maranhão, Brasil.

Figura 3a

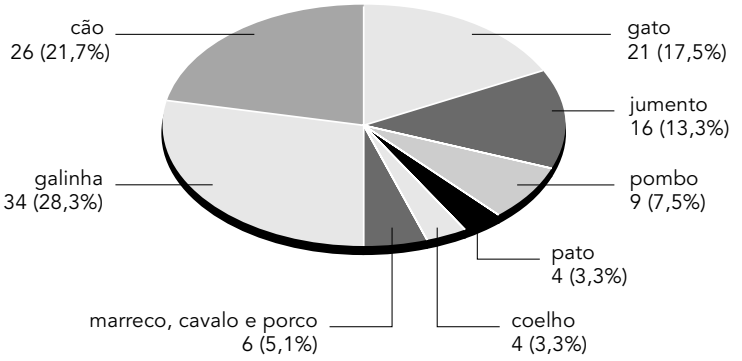

Figura 3b

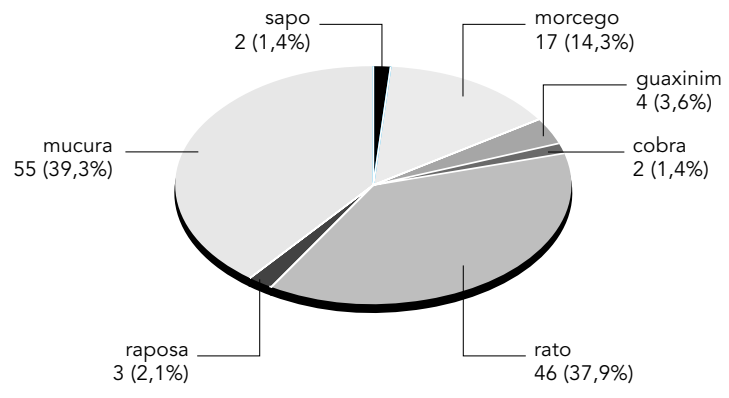

capturaram mais flebótomos sobre galinhas do que em pessoas ou cães.

O papel que a galinha poderia desempenhar na peridomiciliação do L. longipalpis e na epidemiologia do calazar tem sido motivo de reflexão. Na literatura não existe nenhum relato de que aves sejam reservatórios de Leishmania. Ao que consta, são todas refratárias. Nesse caso, a existência de flebotómos infectados, no peridomicílio, dependeria da presença, no mesmo ambiente, de reservatórios sinantrópicos, como a raposa (Alencar et al., 1974/1975; Courtney et al., 1995) e a mucura (Corredor et al., 1989), e de outros animais suscetíveis à infecção por L. chagasi, como o cão, que funcionaria como reservatório doméstico (Alencar, 1959).

De qualquer modo, por mais que não represente uma fonte de infecção para o L. longipalpis, a presença da galinha em grande quantidade no peridomicílio parece constituir o verdadeiro significado epidemiológico, na medida em que funcione como chamariz para o vetor, mantendo-o neste ambiente humano. Este aspecto já ficou comprovado nos estudos realizados nos Municípios da Raposa (Araújo et al., 2000) e de São José de Ribamar (Carvalho et al., 2000), na Ilha de São Luís, quando muitos espécimes do vetor foram capturados no ambiente peridoméstico, utilizando-se a galinha como isca.

O homem apareceu como o terceiro vertebrado mais procurado, sendo superado por ave e roedor, demonstrando o grau de antropofilia do L. longipalpis. Tal hábito já foi observado no nordeste brasileiro (Ward et al., 1983), onde em certas ocasiões o vetor é atraído com mais fre- qüência pelas pessoas do que pelos cães (Deane \& Deane, 1962). Na Costa Rica, Zeledon et al. (1984) também capturaram números significativos de flebótomos em iscas humanas, mas também no cão, porco cavalo e boi. Na Amazônia paraense, por outro lado, o flebótomo está mais inclinado a picar o cão do que as pessoas (Lainson \& Shaw, 1979), todavia, de acordo com Quinnell et al. (1992), o inseto tem hábito alimentar eclético e a atração exercida por determinado hospedeiro seria em função do seu tamanho relativo (um menino, por exemplo, atrai significativamente mais flebótomos do que um cão ou uma galinha e um pouco menos do que seis galinhas).

Os roedores compuseram o grupo de os animais sinantrópicos mais procurados pelos flebótomos, seguidos pela mucura. Ambos foram citados por membros da comunidade de Bom Viver, como os que mais visitam o ambiente peridoméstico, corroborando as observações de Caldas et al. (2001). Este achado reforça a necessidade desses animais serem estudados como possíveis reservatórios da L. chagasi. No decorrer do trabalho, surpreendeu-se uma fêmea de mucura nidificando na cozinha de uma das habitações periféricas em Bom Viver. Evidências epidemiológicas implicam a mucura como reservatório peridomésticos da L. chagasi (Corredor et al., 1989). Ao se confirmar o seu papel como reservatório da Leishmania, na área desse estudo, ela poderia ser considerada um elo entre o ambiente silvestre e o domiciliar.

A distância do galinheiro em relação à residência é um aspecto de grande relevância. Nos 
dois locais de captura dos flebótomos, bem como nas residências cujos moradores foram entrevistados, os abrigos dos animais domésticos distavam no máximo, trinta metros das casas. Tal distância poderia facilitar o acesso do vetor ao intradomicílio, pois está dentro do raio de vôo dos flebótomos (Alexander, 1987; Dye et al., 1991).

A maioria das casas da periferia urbana, guarda muitas semelhanças com o ambiente rural, isto é, são de taipa, não possuem a mínima infra-estrutura sanitária e os abrigos de animais domésticos são muito próximos das habitações. Se isso não bastasse, ainda se encontram galinhas chocas ou com crias, aninhadas dentro das habitações, além de cães e gatos. Em situações como essa, a presença de animais domésticos pode contribuir para aumentar a probabilidade de o vetor adentrar nas habitações e transmitir a infecção intradomiciliarmente.
Em síntese, o encontro de exemplares de $L$. longipalpis alimentados, ao mesmo tempo, com sangue humano, de mucura e do cão no peridomicílio, corrobora a hipótese de que a transmissão da infecção esteja ocorrendo na habitação humana. Teoricamente, todos os elos da cadeia de transmissão da $L$. chagasi estão presentes no ambiente peridoméstico e periurbano do Município de Raposa, o que, em última análise, explica os números de casos que vêm sendo notificados nos últimos anos. Para se ter uma noção da situação, basta verificar que de 1997 a 2001 foram notificados neste Município cerca de 132 casos de calazar, dos quais, 69 eram autóctones da área deste estudo. Neste período os principais acometidos foram crianças de zero a cinco anos. Notem que os casos do Município de Raposa representaram apenas 14,5\% dos registros da Ilha de São Luís, onde está situado este município.

\section{Referências}

ALENCAR, J. E., 1959. Calazar Canino. Contribuição para o Estudo da Epidemiologia do Calazar no Brasil. Tese de Livre Docência. Fortaleza: Imprensa Oficial.

ALENCAR, J. E.; ALMEIDA, Y. M.; SILVA, Z. F.; PAIVA, A. S. \& FONSECA, M. E., 1974/1975. Aspectos atuais do calazar no Ceará. Revista Brasileira de Malariologia e Doenças Tropicais, 26:27-53.

ALEXANDER, B., 1987. Dispersal of Phlebotomine sand flies (Diptera: Psychodidae) in a Colombian coffee plantation. Journal of Medicine and Entomology, 24:552-558.

ALEXANDER, B. \& USMA, M. C., 1994. Potential source of sugar for the phlebotomine sandfly Lutzomyia youngi (Diptera: Psychodidae) in a Colombian coffee plantation. Annals of Tropical Medicine and Parasitology, 88:543-549.

ARAÚJO, J. C.; REBÊLO, J. M. M.; CARVALHO, M. L. \& BARROS, V. L. L., 2000. Composição dos flebotomíneos (Diptera, Psychodidae) do Município da Raposa-MA, Brasil. Área endêmica de leishmanioses. Entomologia y Vectores, 7:33-47. 
AGUIAR, G. M.; VILELA, M. L. \& LIMA, R. B., 1987. Ecology of the sandflies of Itaguai, na area of cutaneous leishmaniasis in the state of Rio de Janeiro. Food preferences (Diptera, Psychodidae, Phlebotominae). Memórias do Instituto Oswaldo Cruz, 82:583-584.

CAMERON, M. M.; PESSOA, F. A.; VASCONCELOS, A. W. \& WARD, R. D., 1995. Sugar meal sources for the phlebotomine sandflies Lutzomyia longipalpis in Ceará State, Brazil. Medicine and Veterinary Entomology, 9:263-272.

CARVALHO, M. L.; REBÊLO, J. M. M.; ARAÚJO, J. C. \& BARROS, V. L. L., 2000. Aspectos ecológicos dos flebotomíneos (Díptera, Psychodidae) do Município de São José de Ribamar, MA, Brasil. Área endêmica de leishmanioses. Entomologia y Vectores, 7:19-32.

CHRISTENSEN, H. A.; ARIAS, J. R.; VASQUEZ, A. M. \& FREITAS, R. A., 1982. Host of sandfly vectors of Leishmania braziliensis guyanensis in the Central Amazon of Brazil. Annals of the Journal of Tropical Medicine and Hygiene, 31:239-242.

CORREDOR, A.; GALLEGO, J. F; TESH, R. B.; PELAEZ, D.; DIAZ, A.; MONTILLA, M. \& PALAU, M. T., 1989. Didelphis marsupialis, na apparent wild reservoir of Leishmania chagasi in Colombia, South America. Transaction of the Royal Society of Tropical Medicine and Hygiene, 83:195.

COURTENAY, O.; MACDONALD, D. W.; LAINSON, R.; SHAW, J. J. \& DYE, C., 1995. Epidemiology of canine leishamaniasis: A comparative sorological study of dogs and foxes in Amazon Brazil. Parasitology, 109:273-279.

CALDAS, A. J. M.; COSTA, J. M. L.; SILVA, A. A. M.; VINHAS, V. \& BARRAL, A., 2001. Risk factors associated with asymptomatic infection by Leishmania chagasi in north-east Brazil. Transactions of the Royal Society of Tropical Medicine and Hygiene, 95:1-8.

DEANE, L. M., 1956. Leishmaniose Visceral no Brasil. Estudos sobre Reservatórios e Transmissores no Estado do Ceará. Tese de Doutorado, Rio de Janeiro: Serviço Nacional de Educação Sanitária.

DEANE, L. M. \& DEANE, M. P., 1962. Visceral leishmaniasis in Brazil: Geographical distribuition and transmission. Revista do Instituto de Medicina Tropical, 4:198-212.

DYE, C.; DAVIES, C. R. \& LAINSON, R., 1991. Communication among phlebotomine sandflies: A field study of domesticated Lutzomyia longipalpis populations in Amazonian Brazil. Animal Behaviour, 42:183-192.
FERREIRA, F. S. C., 1945. A reação das precipitinas aplicadas aos dípteros do gênero Phlebotomus. Anais do Instituto de Medicina Tropical, 2:187-196.

IBGE (Fundação Instituto Brasileiro de Geografia e Estatística), 1996. Contagem Nacional de Populações. Rio de Janeiro: IBGE.

LAINSON, R. \& SHAW, J. J., 1979. The role of animals in the epidemiology of South American leishmaniasis. In: Biology of the Kinetoplastida (E. D. A. Lumsden, ed.), pp. 1-116, London: Academic Press.

MAGNARELLI, L. A. \& MODI, G. B., 1988. Caloric determination of phlebotomine sandflies (Diptera: Psychodidae). Journal of Medicine and Entomology, 20:568-569.

MORRISON, A. C.; FERRO, C.; MORALES, A.; TESH, R. B. \& WILSON, M. L., 1993. Dispersal of the sand fly Lutzomya longipalpis (Diptera: Psychodidae) at an endemic focus of visceral leishmaniasis in Colombia. Journal of Medicine and Entomology, 30:427-435.

QUINNELL, R. J.; DYE, C. \& SHAW, J. J., 1992. Host preferences of the phlebotomine sandfly Lutzomyia longipalpis in Amazonian Brazil. Medical and Veterinary Entomology, 6:195-200.

TESH, R. B.; CHANIOTIS, B.; ARONSON, M. \& JOHNSON, K., 1971. Natural host preferences of Panamanian phelebotomine sand flies as determined by precipitin test. American Journal of Tropical Medicine and Hygiene, 20:150-156.

van HANDEL, E., 1984. Metabolism of nutrients in the adult mosquito. Mosquitoes News, 44:573-579.

WARD, R. D.; RIBEIRO, A. L.; READY, P. D. \& MURTAGH, A., 1983. Reproductive isolation between different forms of Lutzomya longipalpis (Lutz \& Neiva) (Diptera: Psychodidae), the vetor of Leishmania donovani chagasi Cunha \& Chagas and its significance to kala-azar distribution in South America. Memórias do Instituto Oswaldo Cruz, 78:269-280.

ZELEDON, R.; MURILLO, J. \& GUTIERREZ, H., 1984. Observaciones sobre la ecologia de Lutzomya longipalpis (Lutz \& Neiva, 1912) y posibilidades de existencia de leishmaniasis visceral en Costa Rica. Memórias do Instituto Oswaldo Cruz, 79:455-459.

Recebido em 15 de agosto de 2002

Versão final reapresentada em 7 de fevereiro de 2003 Aprovado em 19 de maio de 2003 